\title{
RANDOM QUADRATIC FORMS
}

\author{
JOHN GREGORY AND H. R. HUGHES
}

\begin{abstract}
The results of Boyce for random Sturm-Liouville problems are generalized to random quadratic forms. Order relationships are proved between the means of eigenvalues of a random quadratic form and the eigenvalues of an associated mean quadratic form. Finite-dimensional and infinite-dimensional examples that show these are the best possible results are given. Also included are some results for a general approximation theory for random quadratic forms.
\end{abstract}

\section{INTRODUCTION}

In [1] and [2] Boyce considered the order relationship between the means of eigenvalues for random Sturm-Liouville problems and the corresponding eigenvalues for the associated Sturm-Liouville problem with random coefficients replaced with their means. Subsequently, Kreith [7] modified Boyce's problem to obtain similar results for a stochastic initial value problem of this type.

At first glance these results seem surprising. In the deterministic case, general comparison results exist for the $n$th eigenvalue for pairs of two quadratic forms (see [4] or [5]) and hence Sturm-Liouville problems. But why should any relationship exist if we only average the forms?

The major purpose of this paper is to consider the order relations described above for the $n$th eigenvalue, $n=1,2,3, \ldots$, for quadratic forms. Our setting is that of [5]. Thus, similar results hold for higher-order linear, selfadjoint differential systems and focal or conjugate point theory including quadratic control problems.

The remainder of this paper is as follows. In Section 1 we sketch the deterministic theory needed for the remainder of this paper. In Section 2 we give our main results for order comparison.

In many cases we give counterexamples to show that general results for the $n$th eigenvalue do not always hold. Thus, in Section 3 we consider finitedimensional examples and, in Section 4, infinite-dimensional examples including Sturm-Liouville problems. Finally, in Section 5 we present some new results for a general approximation theory for random quadratic forms.

Received by the editors September 8,1993; originally communicated to the Proceedings of the $A M S$ by Hal L. Smith.

1980 Mathematics Subject Classification (1985 Revision). Primary 34B24; Secondary 34F05, $34 \mathrm{C} 10$.

Key words and phrases. Sturm-Liouville problem, random eigenvalues, continuity of eigenvalues. 


\section{Deterministic THEORY}

Following [4] or [5] we assume that $\mathscr{H}$ is a Hilbert space, $J(x)$ is an elliptic quadratic form, and $K$ is a compact quadratic form defined on $\mathscr{H} . Q(x)$ is a quadratic form if there exists a linear transformation $T$ on $\mathscr{H}$ such that $J(x)=(T x, x)$. The associated bilinear form is $Q(x, y)=(T x, y) . J$ is elliptic if $J$ is equivalent to the inner product on $\mathscr{H}$ except for a subspace of finite co-dimension. $K$ is compact if $K(x)=(T x, x)$ where $T$ is compact. In this setting, a real number $\lambda$ is an eigenvalue of $J(x)$ relative to $K(x)$ if there exists $x \neq 0$ in $\mathscr{H}$, called the eigenvector, such that

$$
J(x, y ; \lambda) \doteq J(x, y)-\lambda K(x, y)=0
$$

for all $y$ in $\mathscr{H}$. We note that the form $J(x ; r)$ is elliptic for any real number $r$.

For an elliptic quadratic form we have two nonnegative integer value functions which correspond, respectively, to the number of negative and zero eigenvalues. Thus we define the signature $s$ of $J(x)$ on a subspace $\mathscr{C} \subset \mathscr{H}$ to be the dimension of a maximal subspace of $\mathscr{C}$ contained in $\{x \in \mathscr{C}: J(x)<$ 0 if $x \neq 0\}$ and the nullity $n$ of $J(x)$ on a subspace $\mathscr{C}$ to be the dimension of the subspace $\{x \in \mathscr{C}: J(x, y)=0$ for all $y \in \mathscr{C}\}$.

If $\mathscr{H}=\mathbb{R}^{n}, A$ is a symmetric matrix, $J(x)=x^{\mathrm{T}} A x$, and $K(x)=x^{\mathrm{T}} x$, then the above definition leads to the usual eigenvalue-eigenvector solutions in $\mathbb{R}^{n}$.

If $\mathscr{H}=\left\{x \in A C(a, b): x^{\prime} \in L^{2}(a, b), \quad x(a)=x(b)=0\right\}, J(x)=$ $\int_{a}^{b}\left[r(t) x^{\prime 2}+q(t) x^{2}\right] d t$, and $K(x)=\int_{a}^{b} p(t) x^{2} d t$, then condition (1) leads to the Sturm-Liouville problem

$$
\left(r x^{\prime}\right)^{\prime}-q x+\lambda p x=0, \quad x(a)=x(b)=0
$$

by using the Euler-Lagrange equation. It is known that this problem has a sequence of eigenvalues $\left\{\lambda_{n}\right\}$ with $\lambda_{1}<\lambda_{2}<\cdots$ and $\lim _{n \rightarrow \infty} \lambda_{n}=\infty$.

We have the following theorem (see [5, p. 111]).

Theorem 1. If $J_{1}(x) \leq J_{2}(x)$ and $K_{1}(x) \geq K_{2}(x)$, then $\lambda_{n}^{(1)} \leq \lambda_{n}^{(2)}$ where $\lambda_{n}^{(i)}$ are the nth eigenvalues of $J_{i}(x)$ relative to $K_{i}(x)$ for $i=1,2$.

In the above Sturm-Liouville problem, the hypothesis holds trivially if $0<$ $r_{1}(t) \leq r_{2}(t), q_{1}(t) \leq q_{2}(t)$, and $p_{1}(t) \geq p_{2}(t)$.

We next turn to a general theory of approximations for the $n$th eigenvalue $\lambda_{n}$. The complete theory is in [5]. Briefly, assume $\Sigma$ is a metric space with metric $\rho$. For each $\sigma$ in $\Sigma$ we assume that $J(x ; \sigma)$ and $K(x ; \sigma)$ are elliptic and compact quadratic forms, respectively, defined on a closed subspace $\mathscr{A}(\sigma)$ of $\mathscr{H}$. In addition, we assume that the relatively weak approximation conditions (1) and (2) in [5, p. 75; 4, pp. 385-386] are satisfied. These conditions hold in all deterministic cases considered below. If we define $s(\lambda, \sigma)$ and $n(\lambda, \sigma)$ to be, respectively, the signature and the nullity of $J(x ; \lambda, \sigma) \doteq J(x ; \sigma)-\lambda K(x ; \sigma)$ on $\mathscr{A}(\sigma)$, then the following result holds.

Theorem 2. For each real $\lambda$, there exists $\delta>0$ such that if $\rho\left(\sigma, \sigma_{0}\right)<\delta$ then

$$
s\left(\sigma_{0}, \lambda\right) \leq s(\sigma, \lambda) \leq s(\sigma, \lambda)+n(\sigma, \lambda) \leq s\left(\sigma_{0}, \lambda\right)+n\left(\sigma_{0}, \lambda\right) .
$$


From [5, p. 110] we also have

Theorem 3. If the nth eigenvalue of $J(\sigma)$ with respect to $K(\sigma), \lambda_{n}(\sigma)$, exists for $\sigma=\sigma_{0}$, it exists in a $\rho$-neighborhood of $\sigma_{0}$ and is a $\rho$-continuous function of $\sigma$.

We note that there is a finite number of negative eigenvalues if we generalize to the condition that $J(x)>0$ whenever $x \neq 0$ and $K(x) \leq 0$.

\section{ORDER RELATIONSHIPS}

We consider here order relationships between the mean values of eigenvalues of a random quadratic form and eigenvalues of the mean quadratic form. This generalizes results in [2].

Let $J(x, y)$ be a symmetric bilinear form and $J(x)$ be the associated quadratic form on $\mathscr{H}$. Let $K(x, y)$ be an inner product on $\mathscr{H}$. Suppose that we have real eigenvalues $\lambda_{j}$ and associated eigenvectors $u_{j} \in \mathscr{H}$ which satisfy

$$
J\left(u_{j}, x\right)-\lambda_{j} K\left(u_{j}, x\right)=0 \quad \text { for every } x \in \mathscr{H} .
$$

Suppose also that the eigenvalues are ordered, $\lambda_{1} \leq \lambda_{2} \leq \cdots$, and the eigenvectors are chosen to be orthonormal with respect to $K$. The following is a generalization of the Rayleigh quotient formula for $\lambda_{1}$ and a related formula in $[3$, p. 459].

Lemma 4. Suppose that the set of eigenvectors $\left\{u_{1}, u_{2}, \ldots\right\}$ is complete; i.e.,

$$
x=\sum_{j} K\left(u_{j}, x\right) u_{j} \quad \text { for all } x \in \mathscr{H} .
$$

Let $\mathscr{I}_{n}$ be the collection of all $K$-orthonormal sets of $n$ elements from $\mathscr{H}$. Then

$$
\sum_{i=1}^{n} \lambda_{i}=\min _{\left\{v_{1}, \ldots, v_{n}\right\} \in \mathcal{F}_{n}} \sum_{i=1}^{n} J\left(v_{i}\right) .
$$

Proof. First we note that $\left\{u_{1}, \ldots, u_{n}\right\} \in \mathscr{I}_{n}$ and $\sum_{i=1}^{n} J\left(u_{i}\right)=\sum_{i=1}^{n} \lambda_{i}$. For general set $\left\{v_{1}, \ldots, v_{n}\right\} \in \mathscr{I}_{n}$ we have

$$
\sum_{i=1}^{n} J\left(v_{i}\right)=\sum_{i=1}^{n} \sum_{j} \lambda_{j}\left[K\left(u_{j}, v_{i}\right)\right]^{2} .
$$

For each integer $i, 1 \leq i \leq n$,

$$
1=K\left(v_{i}, v_{i}\right)=\sum_{j}\left[K\left(u_{j}, v_{i}\right)\right]^{2},
$$

and for each integer $j, j \geq 1$,

$$
1=K\left(u_{j}, u_{j}\right) \geq \sum_{i=1}^{n}\left[K\left(u_{j}, v_{i}\right)\right]^{2} .
$$


Then, defining $K_{j i} \doteq K\left(u_{j}, v_{i}\right)$,

$$
\begin{aligned}
\sum_{i=1}^{n} J\left(v_{i}\right) & =\sum_{i=1}^{n} \sum_{j=1}^{n} \lambda_{j} K_{j i}^{2}+\sum_{i=1}^{n} \sum_{j \geq n+1} \lambda_{j} K_{j i}^{2} \\
& \geq \sum_{i=1}^{n} \sum_{j=1}^{n} \lambda_{j} K_{j i}^{2}+\lambda_{n} \sum_{i=1}^{n} \sum_{j \geq n+1} K_{j i}^{2} \\
& =\sum_{i=1}^{n} \sum_{j=1}^{n} \lambda_{j} K_{j i}^{2}+\lambda_{n} \sum_{i=1}^{n}\left(1-\sum_{j=1}^{n} K_{j i}^{2}\right) \\
& =\sum_{j=1}^{n} \lambda_{j} \sum_{i=1}^{n} K_{j i}^{2}+\lambda_{n} \sum_{j=1}^{n}\left(1-\sum_{i=1}^{n} K_{j i}^{2}\right) \\
& \geq \sum_{j=1}^{n} \lambda_{j}
\end{aligned}
$$

and the conclusion follows.

In the finite-dimensional case, we have an additional similar result for the maximum.

Lemma 5. Suppose $\mathscr{H}$ has dimension $N$. For $n \leq N$, let $\mathscr{I}_{n}$ be the collection of all $K$-orthonormal sets of $n$ elements from $\mathscr{H}$. Then

$$
\sum_{i=N-n+1}^{N} \lambda_{i}=\max _{\left\{v_{1}, \ldots, v_{n}\right\} \in \mathcal{I}_{n}} \sum_{i=1}^{n} J\left(v_{i}\right) .
$$

Let $(\Omega, \mathscr{B}, P)$ be a probability space. We denote the expected value of a random variable $X$ by $\langle X\rangle$. Now we suppose that $J(x ; \omega), \omega \in \Omega$, is a random elliptic quadratic form and $J(x, y ; \omega)$ is the associated random symmetric bilinear form. We let $\lambda_{1}(\omega) \leq \lambda_{2}(\omega) \leq \ldots$ be the ordered eigenvalues of $J(\omega)$ with respect to $K$ for each $\omega \in \Omega$. We shall assume that the corresponding eigenvectors are complete in $\mathscr{H}$. Define the mean bilinear form $\langle J\rangle$ by

$$
\langle J\rangle(x, y) \doteq\langle J(x, y)\rangle \quad \text { for } x, y \in \mathscr{H} .
$$

We assume all expectations exist. Let $\mu_{1} \leq \mu_{2} \leq \ldots$ be the ordered eigenvalues of $\langle J\rangle$. We also assume that the corresponding eigenvectors are complete in $\mathscr{H}$.

Theorem 6. We have the following inequalities:

(i) $\left\langle\lambda_{1}\right\rangle \leq \mu_{1}$, or, more generally,

(ii) $\sum_{i=1}^{n}\left\langle\lambda_{i}\right\rangle \leq \sum_{i=1}^{n} \mu_{i}$.

In addition, if $\mathscr{H}$ is finite-dimensional with dimension $N$,

(iii) $\left\langle\lambda_{N}\right\rangle \geq \mu_{N}$,

or more generally,

(iv) $\sum_{i=n}^{N}\left\langle\lambda_{i}\right\rangle \geq \sum_{i=n}^{N} \mu_{i}$. 
Proof. We prove part (ii) here. The other cases are similar. By Lemma 4, for each $K$-orthonormal set of $n$ vectors $\left\{v_{1}, \ldots, v_{n}\right\}$,

$$
\sum_{i=1}^{n} \lambda_{i}(\omega) \leq \sum_{i=1}^{n} J\left(v_{i} ; \omega\right) \quad \text { for all } \omega \in \Omega
$$

Then

$$
\sum_{i=1}^{n}\left\langle\lambda_{i}\right\rangle \leq \sum_{i=1}^{n}\left\langle J\left(v_{i}\right)\right\rangle=\sum_{i=1}^{n}\langle J\rangle\left(v_{i}\right)
$$

Now

$$
\sum_{i=1}^{n} \mu_{i}=\min _{\left\{v_{1}, \ldots, v_{n}\right\} \in \mathcal{F}_{n}} \sum_{i=1}^{n}\langle J\rangle\left(v_{i}\right),
$$

and thus (ii) follows.

In the $N$-dimensional case, the sum of all eigenvalues satisfies both order relationships and we have

Corollary 7. $\sum_{i=1}^{N}\left\langle\lambda_{i}\right\rangle=\sum_{i=1}^{N} \mu_{i}$.

\section{Finite-Dimensional EXAMPLES}

In this section we study the order relationship between the expected value of the $k$ th eigenvalue and the $k$ th eigenvalue of the expected value in the finitedimensional case. In the previous section we showed that an order relationship can be given for the smallest and largest eigenvalues. We will now show by examples that the intermediate eigenvalues can satisfy either order relationship.

Throughout this section we define for $x$ in $\mathscr{H}=\mathbb{R}^{n}$

$$
J(x)=x^{\mathrm{T}} A x \quad \text { and } \quad K(x)=x^{\mathrm{T}} B x
$$

where $A$ is a symmetric real $n \times n$ matrix and $B$ is a symmetric real positive definite $n \times n$ matrix.

We first give a two-dimensional example with strict inequalities in the smallest and largest eigenvalues.

Example 1. Suppose $\mu_{1} \leq \mu_{2}$ are reals and $\eta$ is a random variable with mean 0 and finite variance. Let

$$
A=\left(\begin{array}{cc}
\mu_{1} & \eta \\
\eta & \mu_{2}
\end{array}\right) \text { and } B=I_{2}=\left(\begin{array}{ll}
1 & 0 \\
0 & 1
\end{array}\right) .
$$

Then the eigenvalues of $\langle J\rangle$ are $\mu_{1}$ and $\mu_{2}$ while the eigenvalues of $J$ are (18)

$$
\lambda_{1}=\frac{\mu_{1}+\mu_{2}}{2}-\sqrt{\frac{\left(\mu_{2}-\mu_{1}\right)^{2}}{4}+\eta^{2}} \text { and } \lambda_{2}=\frac{\mu_{1}+\mu_{2}}{2}+\sqrt{\frac{\left(\mu_{2}-\mu_{1}\right)^{2}}{4}+\eta^{2}} \text {. }
$$

If $\eta \neq 0$ with positive probability, we have the order relationships $\left\langle\lambda_{1}\right\rangle<\mu_{1}$ and $\left\langle\lambda_{2}\right\rangle>\mu_{2}$.

We now consider $n$-dimensional generalizations of the previous example. 
Example 2. Suppose $\mu_{1}<\mu_{2}<\cdots<\mu_{n}$, and let $B=I_{n}$ and

$$
A=\operatorname{diag}\left(\mu_{1}, \mu_{2}, \ldots, \mu_{n}\right)+\left(\begin{array}{cccc}
0 & \eta_{2} & \cdots & \eta_{n} \\
\eta_{2} & 0 & \cdots & 0 \\
\vdots & \vdots & \ddots & \vdots \\
\eta_{n} & 0 & \cdots & 0
\end{array}\right)
$$

where $\eta_{i}$ are mutually independent random variables with mean 0 and $\eta_{i} \neq 0$ with positive probability for each $i$. The characteristic polynomial for this matrix is

$$
Q(\lambda)=\prod_{i=1}^{n}\left(\mu_{i}-\lambda\right)-\sum_{i=2}^{n} \eta_{i}^{2} \prod_{\substack{i=2 \\ j \neq i}}^{n}\left(\mu_{j}-\lambda\right) .
$$

Now for $\lambda$ sufficiently smaller than $\mu_{1}, Q(\lambda)>0$, and for $\lambda$ sufficiently larger than $\mu_{n}, Q(\lambda)$ has the same sign as $(-1)^{n}$. Evaluating at each $\mu_{i}$, we have $Q\left(\mu_{1}\right) \leq 0, Q\left(\mu_{2}\right) \leq 0$, and $Q\left(\mu_{i}\right)$ alternate $\leq 0, \geq 0$ for $i \geq 2$. Furthermore, with positive probability, all of these inequalities hold strictly. It follows that with positive probability, $\lambda_{1}<\mu_{1}$ and $\lambda_{i}>\mu_{i}$ for $i \geq 2$. Taking expectations, the mean eigenvalues also satisfy these relationships.

In a similar way, let $B=I_{n}$ and

$$
A=\operatorname{diag}\left(\mu_{1}, \mu_{2}, \ldots, \mu_{n}\right)+\left(\begin{array}{cccc}
0 & \cdots & 0 & \eta_{1} \\
\vdots & \ddots & \vdots & \vdots \\
0 & \cdots & 0 & \eta_{n-1} \\
\eta_{1} & \cdots & \eta_{n-1} & 0
\end{array}\right)
$$

where $\eta_{i}$ are mutually independent random variables with mean 0 and $\eta_{i} \neq 0$ with positive probability for each $i$. Then we have $\left\langle\lambda_{i}\right\rangle<\mu_{i}$ for $1 \leq i \leq n-1$ and $\left\langle\lambda_{n}\right\rangle>\mu_{n}$.

Example 3. We can construct examples where the intermediate eigenvalues satisfy either order relationship by using the matrices in Examples 1 and 2 as blocks of a larger matrix.

Allowing $A$ and $B$ both to be random, we can construct examples where any eigenvalue can satisfy either order relationship.

Example 4. Suppose $\mu_{1}<\mu_{2}<\cdots<\mu_{n}$. Let $A=\operatorname{diag}\left(\eta_{1}(\omega), \ldots, \eta_{n}(\omega)\right)$ where $\left\langle\eta_{i}\right\rangle=\mu_{i}$ for $1 \leq i \leq n$, and let $B=\operatorname{diag}\left(\kappa_{1}(\omega), \ldots, \kappa_{n}(\omega)\right)$ where $\left\langle\kappa_{i}\right\rangle=1$ for $1 \leq i \leq n$. Then the eigenvalues of $\langle J\rangle$ relative to $\langle K\rangle$ are $\mu_{1}, \ldots, \mu_{n}$ and the (random) eigenvalues of $J$ relative to $K$ are $\eta_{i} / \kappa_{i}$ for $1 \leq i \leq n$. Let $\Omega=\left\{\omega_{1}, \omega_{2}\right\}$ and $P\left(\omega_{1}\right)=1 / 2=P\left(\omega_{2}\right)$. Now if we take

$$
\begin{array}{ll}
\eta_{i}\left(\omega_{1}\right)=\mu_{i}\left(1-\delta_{i}\right), & \eta_{i}\left(\omega_{2}\right)=\mu_{i}\left(1+\delta_{i}\right), \\
\kappa_{i}\left(\omega_{1}\right)=1-\epsilon_{i}, & \kappa_{i}\left(\omega_{2}\right)=1+\epsilon_{i},
\end{array}
$$

we have for sufficiently small positive $\epsilon_{i}$ and $\delta_{i}$,

$$
\left\langle\lambda_{i}\right\rangle=\left\langle\frac{\eta_{i}}{\kappa_{i}}\right\rangle=\frac{\mu_{i}\left(1-\epsilon_{i} \delta_{i}\right)}{1-\epsilon_{i}^{2}} \quad \begin{cases}>\mu_{i} & \text { if } 0<\delta_{i}<\epsilon_{i}, \\ <\mu_{i} & \text { if } 0<\epsilon_{i}<\delta_{i} .\end{cases}
$$




\section{INFINITE-DIMENSIONAL EXAMPLES}

The theory of quadratic forms on infinite-dimensional spaces includes, for example, the Sturm-Liouville theory. For general quadratic forms on infinitedimensional spaces we get results very similar to those of the preceding section.

Example 5. We consider the eigenvalue problem where $J$ is random and $K$ is deterministic. We can construct examples where the order relations between the eigenvalues of the mean quadratic form, $\mu_{i}$, and the mean eigenvalues of the random quadratic form, $\left\langle\lambda_{i}\right\rangle$, are specified in either direction for a finite number of them not including the first. We do this by applying the ideas of Example 3 on a finite-dimensional subspace of $\mathscr{H}$.

It is not clear whether Sturm-Liouville boundary value problem examples can be constructed for the quadratic forms in Example 5. However we give the quadratic form version of a similar Sturm-Liouville example of Boyce [2].

Example 6. Let $\mathscr{H}=\left\{x \in A C(0, \pi): x^{\prime} \in L^{2}(0, \pi), \quad x(0)=x(\pi)=0\right\}$,

$$
J(x, \omega)=\int_{0}^{\pi}\left[x^{\prime 2}+(1+\epsilon \eta(\omega) \cos t) x^{2}\right] d t, \quad \text { and } \quad K(x)=\int_{0}^{\pi} x^{2} d t,
$$

where $\langle\eta\rangle=0, \eta$ is bounded a.s., and $\eta \neq 0$ with positive probability. Boyce shows that for sufficiently small $\epsilon>0,\left\langle\lambda_{k}\right\rangle>\mu_{k}=1+k^{2}$ for $k \geq 2$.

As in the finite-dimensional case, allowing $K$ also to be random allows for more diversity in the order relationships. Consider the following SturmLiouville example.

Example 7. Let $\mathscr{H}$ be as before, and let

$$
J(x, \omega)=\int_{0}^{\pi}\left[p(\omega) x^{\prime 2}+q(\omega) x^{2}\right] d t \text { and } K(x, \omega)=\int_{0}^{\pi} r(\omega) x^{2} d t
$$

where $p>0, r>0, q>-p,\langle p\rangle=1,\langle q\rangle=0$, and $\langle r\rangle=1$. Then the eigenvalues of $\langle J\rangle$ relative to $\langle K\rangle$ are $\mu_{k}=k^{2}$ and the random eigenvalues of $J$ relative to $K$ are

$$
\lambda_{k}(\omega)=\frac{p(\omega) k^{2}+q(\omega)}{r(\omega)} .
$$

In particular, let $\Omega=\left\{\omega_{1}, \omega_{2}\right\}$ and $P\left(\omega_{1}\right)=1 / 2=P\left(\omega_{2}\right)$. If

$$
\begin{array}{lll}
p\left(\omega_{1}\right)=1+\epsilon, & p\left(\omega_{2}\right)=1-\epsilon, \\
q\left(\omega_{1}\right)=-2 n^{2} \epsilon, & q\left(\omega_{2}\right)=2 n^{2} \epsilon, \\
r\left(\omega_{1}\right)=1-\epsilon, & r\left(\omega_{2}\right)=1+\epsilon,
\end{array}
$$

then, for sufficiently small $\epsilon>0$,

$$
\left\langle\lambda_{k}\right\rangle=k^{2}+\frac{2 \epsilon^{2}\left(k^{2}-n^{2}\right)}{1-\epsilon^{2}}
$$

and thus

$$
\left\langle\lambda_{k}\right\rangle \begin{cases}<\mu_{k} & \text { if } k<n, \\ >\mu_{k} & \text { if } k>n .\end{cases}
$$


On the other hand, if

$$
\begin{array}{ll}
p\left(\omega_{1}\right)=1-2 \epsilon, & p\left(\omega_{2}\right)=1+2 \epsilon, \\
q\left(\omega_{1}\right)=n^{2} \epsilon, & q\left(\omega_{2}\right)=-n^{2} \epsilon, \\
r\left(\omega_{1}\right)=1-\epsilon, & r\left(\omega_{2}\right)=1+\epsilon,
\end{array}
$$

then, for sufficiently small $\epsilon>0$,

$$
\left\langle\lambda_{k}\right\rangle=k^{2}+\frac{\epsilon^{2}\left(n^{2}-k^{2}\right)}{1-\epsilon^{2}}
$$

and thus

$$
\left\langle\lambda_{k}\right\rangle \begin{cases}>\mu_{k} & \text { if } k<n \\ <\mu_{k} & \text { if } k>n\end{cases}
$$

\section{APPROXIMATION THEORY}

Let $\Sigma$ be a metric space with metric $\rho$ and $J(x ; \sigma), \sigma \in \Sigma$, be a family of quadratic forms for which the conclusion of Theorem 2 holds. That is, for each $\lambda$, there exists $\delta>0$ such that if $\rho\left(\sigma, \sigma_{0}\right)<\delta$ then the inequaltities of (3) hold. Then it follows immediately that if $\eta$ is a $\Sigma$-valued random variable such that $\rho\left(\eta, \sigma_{0}\right)<\delta$, a.s., then

$$
s\left(\sigma_{0}, \lambda\right) \leq s(\eta, \lambda) \leq s(\eta, \lambda)+n(\eta, \lambda) \leq s\left(\sigma_{0}, \lambda\right)+n\left(\sigma_{0}, \lambda\right), \quad \text { a.s. }
$$

We define $\bar{s}(\eta, \lambda)$ to be the number of mean eigenvalues, $\left\langle\lambda_{k}(\eta)\right\rangle$, less than $\lambda$ and $\bar{n}(\eta, \lambda)$ to be the number of mean eigenvalues equal to $\lambda$. Then we have the following approximation theorem for the mean eigenvalues.

Theorem 8. Let $\lambda$ be fixed, and suppose that the conclusion of Theorem 2 holds for $J(x ; \sigma)$. Then for the same $\delta>0$, if $\rho\left(\eta, \sigma_{0}\right)<\delta$, a.s.,

$$
s\left(\sigma_{0}, \lambda\right) \leq \bar{s}(\eta, \lambda) \leq \bar{s}(\eta, \lambda)+\bar{n}(\eta, \lambda) \leq s\left(\sigma_{0}, \lambda\right)+n\left(\sigma_{0}, \lambda\right) .
$$

Proof. Suppose $s\left(\sigma_{0}, \lambda\right)=m$. Then by (33), if $\rho\left(\eta, \sigma_{0}\right)<\delta$, a.s., $s(\eta, \lambda) \geq$ $m$, a.s. Thus $\lambda_{m}(\eta)<\lambda$, a.s., and so $\langle\lambda(\eta)\rangle<\lambda$, and it follows that $\bar{s}(\eta, \lambda) \geq$ $m=s\left(\sigma_{0}, \lambda\right)$. The last inequality follows in a similar way, noting that $s(\eta, \lambda)+$ $n(\eta, \lambda) \leq M$ only if $\lambda_{M+1}(\eta)>\lambda$ and the converse holds for $\bar{s}+\bar{n}$.

In certain cases, we can get approximation results with less stringent hypotheses.

Example 1 revisited. Consider Example 1 with the added condition that $\operatorname{Var}(\eta)$ $<\delta^{2}$. Since

$$
\frac{\mu_{2}-\mu_{1}}{2} \leq \sqrt{\frac{\left(\mu_{2}-\mu_{1}\right)^{2}}{4}+\eta^{2}} \leq \frac{\mu_{2}-\mu_{1}}{2}+|\eta|,
$$

we have that

$$
\mu_{1}-|\eta| \leq \lambda_{1} \leq \mu_{1} \quad \text { and } \quad \mu_{2} \leq \lambda_{2} \leq \mu_{2}+|\eta| .
$$

Thus $\lambda_{i}$ approximates $\mu_{i}$ in a mean-square sense:

$$
\left\langle\left(\lambda_{i}-\mu_{i}\right)^{2}\right\rangle^{1 / 2}<\delta, \quad i=1,2 .
$$


It follows also from this that

$$
\left|\left\langle\lambda_{i}\right\rangle-\mu_{i}\right|<\delta, \quad i=1,2,
$$

and thus for each $\lambda$ there exists a $\delta>0$ such that if $\operatorname{Var}(\eta)<\delta^{2},(34)$ holds. Thus we get the approximation result for the mean eigenvalue without requiring a uniform bound on $\eta$.

The authors would like to thank Professor Keith Kreith for calling this interesting topic to our attention.

\section{REFERENCES}

1. W. E. Boyce, Random eigenvalue problems, Probabilistic Methods in Applied Mathematics (A. T. Bharucha-Reid, ed.), Academic Press, New York, 1968, pp. 1-73.

2. _ On a conjecture concerning the means of the eigenvalues of random SturmLiouville boundary value problems, Quart. Appl. Math. 38 (1980), 241-245.

3. R. Courant and D. Hilbert, Methods of mathematical physics, vol. 1, Interscience, New York, 1953.

4. J. Gregory, An approximation theory for elliptic quadratic forms on Hilbert spaces: application to the eigenvalue problem for compact quadratic forms, Pacific J. Math. 37 (1970), 383-395.

5. __, Quadratic form theory and differential equations, Math. Sci. Engr., vol. 152, Academic Press, San Diego, CA, 1980.

6. M. R. Hestenes, Applications of the theory of quadratic forms in Hilbert space in the calculus of variations, Pacific J. Math. 1 (1951), 525-582.

7. K. Kreith, Lower estimates for zeros of stochastic Sturm-Liouville problems, Proc. Amer. Math. Soc. 92 (1984), 515-518.

Department of Mathematics, Southern Illinois University at Carbondale, CarbonDALE, ILLINOIS 62901

E-mail address: hrhughes@c-math1. siu.edu 